\title{
A letter from Professor Samuel J. Danishefsky
}

\author{
Samuel J. Danishefsky ${ }^{1,2}$
}

Received: 8 March 2019 / Accepted: 13 March 2019 / Published online: 27 May 2019

(c) The Author(s), under exclusive licence to the Japan Antibiotics Research Association 2019

\section{Dear Editors,}

Let me express how thankful I am for your more than generous gesture in inviting publications from my research associates and for combining them in a single issue of the Journal of Antibiotics. It may be of interest for you to know that the Journal of Antibiotics was actually the third journal to which I personally subscribed. You'll understand, that the first two were the JACS and the JOC. These were staples for researchers in North America, bringing forth a broad variety of issues in chemistry as a whole, and particularly in organic chemistry.

When it came time to expand my personal holdings, the next journal to which I turned, was the Journal of Antibiotics. This action followed the first trip I made to Japan in 1980, where I was a fellow (sponsored by Professor Teruaki Mukaiyama) of the Japan Society for the Promotion of Science. Indeed, I used part of my honorarium from that visit to become a proud subscriber of the Journal of Antibiotics. I did so because more than any other publication, the Journal of Antibiotics spoke to my developing passion for natural products. Eagerly, I would particularly await the publication date of this journal and upon its arrival, I would lunge into it, thereby beholding the fantastic universe of small molecule biologically active natural products.

My interest here was at two levels. First there was the pure joy of gazing upon the wonderful architectural audacity that nature endowed to plants, fungi, corals, and bacteria. These small molecule natural products were at that time, referred to as secondary metabolites. This terminology

Samuel J. Danishefsky

danishes@mskcc.org

1 Emeritus, Columbia University, New York, United States of America

2 Emeritus, Memorial Sloan-Kettering Cancer Center, New York, United States of America (which I found somewhat dismissive) reflected the truth that the major players at the chemistry-biology interface were carbohydrates, proteins, and nucleic acids. However, living in the more insular community of organic chemists, these the Journal of Antibiotics entries were for me, primary rather than secondary. In addition to being potentially useful agents in medicine, many of these small molecule natural products implicitly posed challenges to the creative tendencies of organic chemists, operating in the exciting universe of total synthesis.

Moreover, since these structures appeared in the Journal of Antibiotics, almost by definition, they would be endowed with biological activity, which at least in theory, could be harnessed for human good. Hence another motive in devouring each incoming issue of the Journal of Antibiotics was the possibility of finding structures and biological motifs which could spark programs in our laboratories, (and potentially enrich the quality of research proposals to various funding instruments). Furthermore, by following up suggestive references in your journal, I was further exposed to an even broader menu of potential challenges. Among these molecules presented, courtesy of the Journal of Antibiotics, which became part of our continuing research programs, were coriolin (actually first synthesized by Professor Tatsuta and co-workers), calicheamicin, dynemicin, ardeemin, tryprostatins, compactin, prostaglandins, epothilones, taxanes, eleutherobin, mitomycins, pentalenolactone, and migrastatin. In each of these cases, we would start and complete a program directed to a total synthesis of the "wild type" structure. In these thrusts, we would always make a good faith effort to realize new ideas in synthetic architecture and methodology, appropriate to the target. In some select cases, having achieved the total synthesis, we brought to bear a concept which we called molecular editing. Previously the standard approach of using natural products in medicinal chemistry was to start with nature's "wild type", generally available by isolation, and then affect molecular modifications based upon doable chemistry (or enzymatic methodology). In our view, synthesis offered a way to implement molecular modifications which could not be 
realized, by operating on the "wild type" itself due to limitations in the required chemistry. Rather it was our hope to use total synthesis to come close enough to the actual structure, still allowing for divergence. There would thus be enabled structural modifications, not obtainable from the "wild type" natural product itself. (We note that this pursuit was anticipated by the pioneering research of Professor E.J. Corey, in the prostaglandin area.) Some of these "total synthesis derived mutants" did indeed serve to improve upon biological performance, at the level of drug availability, toxicity abatement, and even potency.

It was in the latter part of my career, that I began to feel a compulsion to attack synthetic problems, associated with the "larger molecule main drivers" of biological life. Struggle as I did, I was unable to conceive as to how I might be able to offer valuable contributions to the synthesis of nucleic acids. All of my conjectures in this field seemed to suffer from being too little and too late. By contrast, the other two primary biological drivers were less mature in terms of access by chemical synthesis. I dared to believe that they might benefit by intervention of my ambitious associates.

The first of these expeditions was in the synthesis of complex oligosaccharides. I should note that our involvement in this area, involved a measure of happenstance. Allow me to explain. Our earlier interest in small molecule total synthesis led us to treasure (and possibly even to improve upon) the potentialities of the hallowed Diels Alder reaction. One such important extension involved the generation of new and synergistically activated 1, 3substituted dienes. Here I would particularly note the critical role of my esteemed colleague, Dr. Takeshi Kitahara, then a newly arrived post-doctoral from the laboratory of Professors Masanao Matsui and Kenji Mori. Having demonstrated the value of these new dienes in cycloaddition reactions with carbon-carbon dienophiles, one came to wonder about their possible extension to hetero dienophiles. Happily, a young graduate student (James Kerwin) found that under Lewis acid catalysis, our synergistically activated dienes underwent smooth cycloaddition with a broad range of carbon-oxygen double bonds of aldehydes and carbon-nitrogen double bonds of imines. In the case of the aldehydes, this provided us with dihydropyrones. Upon reduction of the keto group of the product (really a vinylogous ester) we had stumbled upon fully synthetic glycals with complete control of functionality at C-4 and C-6. In our early work, we sought to endow this chemistry with the added feature of enantiotopic control. In the event we were then able to achieve significant enantiotopic enrichments in the cycloaddition reaction. We can't help but note that these were perhaps the first cases of catalytically induced substantial (50-60\%) enantiotopic control in the formation of carbon-carbon bonds. While this progress, on the E.E. front, was certainly pleasing, it was clearly insufficient for broad scale application when such control was needed. Happily, as a consequence of groundbreaking research of Professor Eric Jacobsen at Harvard, this problem too has been satisfactorily solved. In this way, we could gain access to potential building blocks to fashion unnatural oligosaccharides in a few powerful steps. However, we first focused on dealing with the problem of reaching "wild type" oligosaccharides by total synthesis. Here we began with glycosyl donors arising from natural type sugar precursors. We were able to accomplish new strategies for solution-based glycosylation even realizing extension to the solid phase oligosaccharide synthesis. I recall, with no small measure of satisfaction that my colleagues accomplished the most advanced examples of complex (hexasaccharides!) glycans, by solid phase synthesis known at that time.

Continuing our explorations of the carbohydrate landscape, we came to learn that often oligosaccharides appear in the context of their attachment to polypeptides, and proteins. Here again, synthesizing such molecules in a chemical laboratory seemed to constitute a challenge worthy of the attention of my eager, young, and ambitious colleagues. We reasoned that if we were to be successful, we must first engage in issues associated with the synthesis of non-glycosylated small proteins. Indeed, my colleagues fashioned new methods to achieve chemically based ligations of smaller peptide building blocks enroute to more formidable targets. Having found success in this area, and having gained skills in oligosaccharide synthesis, we were ready to engage a virtually neglected challenge, i.e.; generating complex glycopolypeptides and small glycoproteins by chemical means. Again, my incredibly dedicated colleagues managed to achieve major field setting advances in this area.

These successes enabled us to test the terrain that might eventually lead to the possibility of reaching vaccines derived by exclusive chemical means. In so doing, we were anticipating (albeit in rather primitive form) a vision of the future which seeks to marshal immunotherapeutic strategies for the life and death struggle against cancer. Following the efforts of incredibly dedicated young men and women, we actually brought to clinical trial the first cases of synthetically derived vaccines. One of these (the globo- $\mathrm{H}$ vaccine) showed the hoped-for immunological responses in a Phase-I clinical trial directed to breast cancer. Moreover, it was taken all the way through a phase III expansion.

It was further reasoned that the efficacy of vaccination in a cancer setting could be enhanced if it enabled immunological reaction which covers a broader waterfront of tumor related antigens. It is in this spirit that our secondgeneration vaccine (see image, Fig. 1), was created, in collaboration with the Organic Synthesis Core laboratory, 


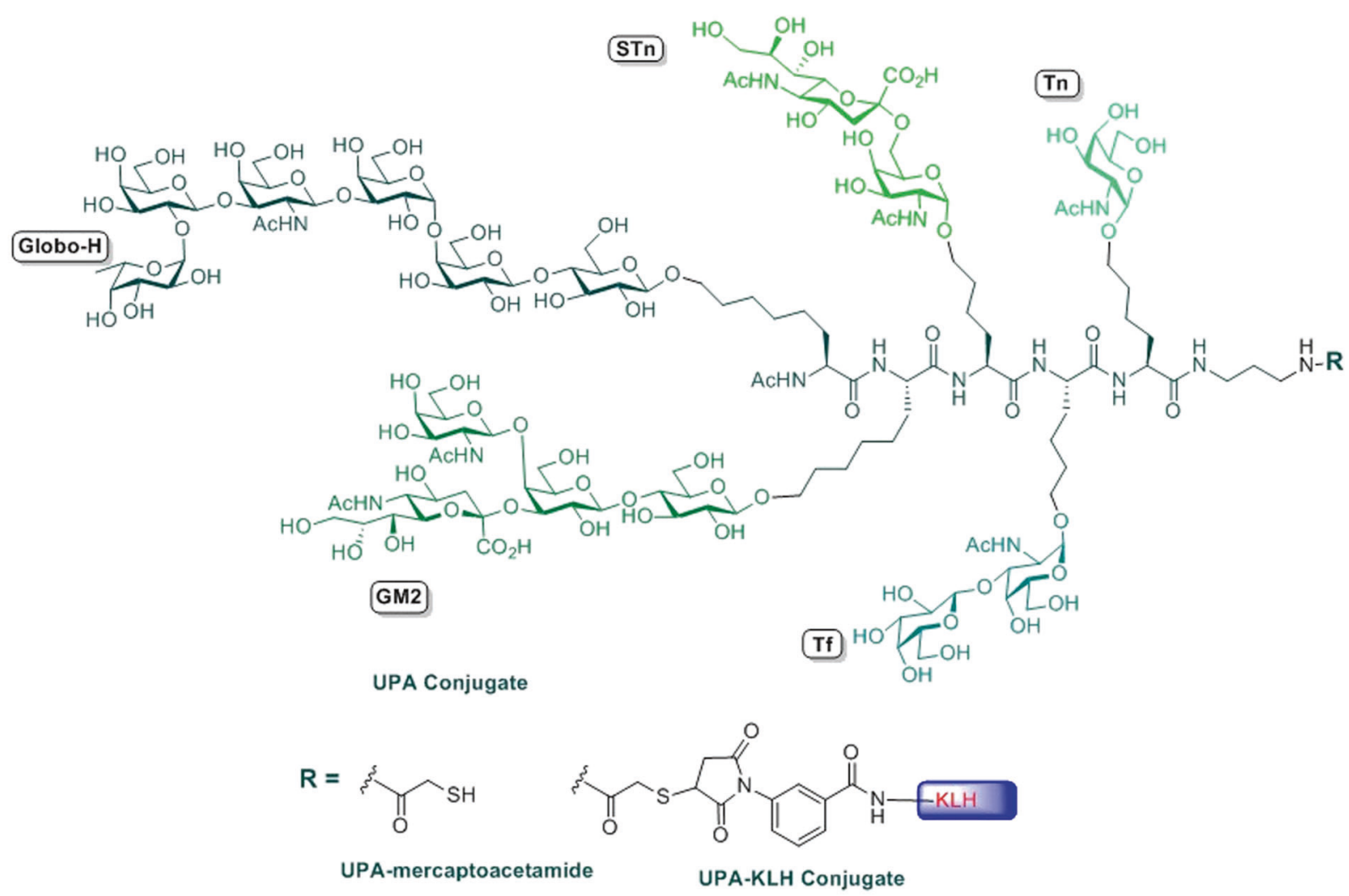

Fig. 1 Structure of the ovarian cancer vaccine construct

headed by Dr. Ouathek Ouerfelli. As seen, it contains a multiplicity of potentially relevant anti-cancer carbohydrate-based antigens, in a single molecule. This amazing vaccine was brought forth the required Good Manufacturing Practices (GMP)-state to a Phase-I ovarian cancer trial. The results pointed to a promising antibody producing outcome in a recently completed trial at Memorial Sloan-Kettering Hospital. In this way we have avoided the need for a cocktail of individual vaccines. Such mixtures would obviously pose serious regulatory issues required for vaccine approval.

Finally, at the very end of our operations in collaboration with Dr. Baptiste Aussedat and Dr. William Walkowicz, of the Sloan-Kettering Institute, and with Professor Barton Haynes at Duke University, we were able to fashion fully synthetic vaccines of promising immunological value in the prevention of the dreaded disease of AIDS. Thus, in retrospect, it was the love of small molecule natural products, enhanced by your journal, that in effect, prompted the "big molecule" phase of my research career. It is for these reasons and many others, that I feel a continuing attachment and gratitude to the Journal of Antibiotics.

It goes without saying that none of the endeavors described above, would have been possible, without the active collaboration of a remarkable group of colleagues.
Upon arrival at the University of Pittsburgh, I did have some opportunities to conduct personal experiments, but only for the first 2-3 years. Even during that period, and certainly thereafter, all of the science which we were fortunate enough to achieve, was really a collaborative enterprise with a unique group of young men and women, undergraduates, graduate students, and post-doctorals. It is their dedication, creativity, experimental skills, and inspiration, which made everything possible.

Of course, one hopes that these accomplishments might prove to be even more valuable with the passage of time. The longing that one's discoveries will have long term consequences is part of the DNA of every scientist. However, the larger truth is that as time progresses, what was considered to be a striking new insight in 1964, is likely to be regarded as an esoteric footnote in 2020.

Alas there is relief from the dread of obsolescence! That redemption is rooted in the human legacy that one leaves behind. Happily, I have been particularly blessed with unusual colleagues, who have gone on to exciting careers of their own. My hope is that they may have benefitted at least in some small measure, from the time they spent under our roof. I say "our" because of the unique contributions of Dr. Sarah Danishefsky. It is together, that we charted this long journey to afford both a laboratory and a home for aspiring 
scientists. Together, Sarah and I formed a lifetime partnership, welcoming generations of remarkable youngsters to our midst. Many of these colleagues were kind enough to provide contributions to this issue of the Journal of Antibiotics. To them, and to all the others, I extend heartfelt thanks and a personal blessing. May their lives be enriched by wonderful families, good health, good fortune and the deep satisfaction of creative pursuit of difficult but worthy goals directed toward human good.

With all the best wishes!

Sincerely yours,

Samuel J. Danishefsky 